\title{
Does Hydrotherapy Help or Hinder Adaptation to Training in Competitive Cyclists?
}

\author{
Shona L Halson ${ }^{1}$, Jason Bartram ${ }^{1}$, Nicholas West ${ }^{1,2}$, Jessica Stephens ${ }^{1}$, \\ Christos K Argus ${ }^{1}$, Matthew W Driller ${ }^{1}$, Charli Sargent ${ }^{3}$, Michele Lastella ${ }^{3}$, \\ Will G. Hopkins ${ }^{4}$ and David T Martin ${ }^{1}$
}

\footnotetext{
${ }^{1}$ AIS Physiology, Australian Institute of Sport, PO Box 176, Belconnen, ACT, Australia

${ }^{2}$ School of Medicine, Griffith Health Institute, Griffith University, Gold Coast, Australia

${ }^{3}$ Appleton Institute for Behavioural Science, Central Queensland University, Adelaide, Australia

${ }^{4}$ Sport Performance Research Institute, Auckland University of Technology, Auckland, New Zealand
}

Address for Correspondence:

Shona L Halson

Department of Physiology

Australian Institute of Sport

PO Box 176, Belconnen ACT, Australia, 2616

Phone: (+61) 0262141589

Fax: $\quad(+61) 0262141836$

Email: shona.halson@ausport.gov.au

Running Title: Hydrotherapy in Competitive Cyclists

This investigation was supported by funding from the Australian Sports Commission.

The authors declare that there are no conflicts of interest in undertaking this study. 


\begin{abstract}
Purpose Cold water immersion may be beneficial for acute recovery from exercise, but it may impair long-term performance by attenuating the stimuli responsible for adaptation to training. We compared effects of cold water immersion and passive rest on cycling performance during a simulated cycling grand tour.
\end{abstract}

Methods Thirty-four male endurance-trained competitive cyclists were randomized to cold water immersion (CWI) for four times per week for 15 min at $15^{\circ} \mathrm{C}$ or control (passive recovery) groups for $7 \mathrm{~d}$ of baseline training, $21 \mathrm{~d}$ of intensified training, and an $11 \mathrm{~d}$ taper. Criteria for completion of training and testing were satisfied by 10 cyclists in the CWI group (maximal aerobic power, $5.13 \pm 0.21 \mathrm{~W} / \mathrm{kg}$; mean $\pm \mathrm{SD}$ ) and 11 in the control group (5.01 \pm $0.41 \mathrm{~W} / \mathrm{kg}$ ). Each week cyclists completed a high intensity interval cycling test and two 4min bouts separated by $30 \mathrm{~min}$. CWI was performed four times per week for $15 \mathrm{~min}$ at $15^{\circ} \mathrm{C}$.

Results Between baseline and taper, cyclists in the CWI group had an unclear change in overall 4 -min power relative to control $(2.7 \%, \pm 5.7 \%)$, although mean power in the second effort relative to the first was likely higher for the CWI group relative to control $(3.0 \%$, $\pm 3.8 \%$ ). The change in 1-s maximum mean sprint power in the CWI group was likely beneficial compared to control $(4.4 \%, \pm 4.2 \%)$. Differences between groups for the 10 -min time trial were unclear $(-0.4 \%, \pm 4.3 \%)$.

Conclusion While some effects of CWI on performance were unclear, data from this study do not support recent speculation that CWI is detrimental to performance following increased training load in competitive cyclists.

Keywords: Cold Water Immersion, Adaptation, Cycling, Recovery, Performance 


\section{INTRODUCTION}

Paragraph Number 1Recovery research, specifically hydrotherapy research, has gained significant attention in recent years, with a number of studies documenting acute performance benefits following hydrotherapy (9). However, whether hydrotherapy, in particular cold water immersion, enhances or reduces adaptation to training has become a topic of interest. Training theory suggests that fatigue and/or inflammation post exercise is necessary to promote long-term adaptations to training and subsequent improvements in performance. Based on the improvements in performance observed in acute cold water immersion studies, two adaptation theories have been proposed. The first is that hydrotherapy allows athletes to perform subsequent training sessions with a greater training load or quality, thus resulting in an enhanced stimulus for adaptation. Conversely, the second theory suggests that cold water immersion may decrease adaptations to training due to minimization of fatigue and inflammation occurring following training. Thus, some practitioners are questioning the use of chronic hydrotherapy treatment in elite athletes with respect to a potential negative influence on training adaptation.

Paragraph Number 2Much of the debate has arisen following preliminary hydrotherapy studies with varying performance assessments and/or biochemical and inflammatory marker measurements $(3,5,24)$ or studies using ice or cold packs (13). Yamane et al (24), reported that regular cold water immersion resulted in an attenuation of cycling performance improvements when compared to a control condition over a 4-6 week training period. Similar findings were reported by Higgins et al. (3) whereby repeat sprint test performance decreased with cold water immersion when compared with control in amateur rugby union players. 
Well-controlled studies which investigate the role of cold water immersion on long-term adaptations and performance are warranted. Previous research does not enable wide-spread conclusions regarding the suitability of chronic hydrotherapy recovery protocols for elite athletes. Further research is necessary to address fundamental recovery questions for elite athletes and coaches. Therefore, the purpose of the present investigation was to examine the effects of cold water immersion, using contemporary methodology on cycling performance over a 39-day training block.

\section{METHODS}

\section{Subjects}

Paragraph Number 3Thirty-four endurance trained cyclists were initially recruited for this study. All participants were at a nationally competitive level and were in an early phase of their domestic season. In the initial week of the study the subjects were divided into two groups; recovery (REC) and control (CONT). REC and CONT groups were matched based on subjects maximal aerobic power (MAP) (w/kg) and their belief in whether recovery would help or hinder adaptation to the training block (rated from 0-100).

Paragraph Number 4Twenty-one cyclists completed all the training and testing requirements without illness and/or injury. The final twenty-one cyclists consisted of ten subjects from the REC group (age: $20.2 \mathrm{yr} \pm 1.7$; MAP: $5.13 \mathrm{~W} / \mathrm{kg} \pm 0.21$; Mass: $70.9 \mathrm{~kg} \pm 6.5$ ), and eleven subjects from the CONT group (age: $19.8 \mathrm{yr} \pm 1.7$; MAP: $5.01 \mathrm{~W} / \mathrm{kg} \pm 0.41$, Mass: $68.9 \mathrm{~kg} \pm$ 8.0). All participants were informed of the methods, procedures and risks of the study and provided written informed consent prior to taking part. This study was approved by the Australian Institute of Sport Research Ethics Committee. 


\section{Experimental design}

\section{Training}

Paragraph Number 5Subjects completed 39-days of structured training consisting of a mixture of low-moderate intensity road rides and high intensity interval sessions completed on ergometers in a laboratory. The first seven days was considered a baseline block and consisted of a low volume of hours spent road cycling, as well as both familiarisation and baseline ergometer tests (a high intensity interval set (HIIT) and a 2x4 minute maximal time trial $\left(2 \mathrm{xMMP}_{4 \mathrm{~min}}\right)$; each described below). Also included in the first week was an incremental step test to exhaustion (100W initial increasing 50W every 5 min until exhaustion) to assess maximal aerobic power (MAP). The next twenty-one days of the training block was spent completing increasingly weekly load via longer duration road rides. Three interval sets were also completed each week, HIIT, $2 \mathrm{xMMP}_{4 \min }$ and a third high-intensity interval training set (Set 3). In the final eleven days the overall training duration was reduced extensively to allow a taper period. The HIIT, $2 \mathrm{xMMP}_{4 \min }$ and Set3 interval sets were all completed twice throughout the taper period (see Table 1).

\section{Recovery Intervention}

Paragraph Number 6Throughout the thirty-nine days of training the REC group performed four sessions of cold water immersion (CWI) per week, in which subjects submerged their body (excluding head and neck). Each supervised recovery session involved 15 minutes of $\mathrm{CWI}$ at $15.3 \pm 0.3^{\circ} \mathrm{C}$ completed post training or testing (within 30 minutes). Subjects were not allowed to shower immediately post CWI, but were able to immediately towel dry and change into dry clothing. Recovery sessions were completed at the end of the baseline phase, at the end of each week during the intensified training phase and at the end of each week of the taper phase (See Table 1). The four days were scheduled to start one day prior to the HIIT 
interval set for consistency regarding the performance measures. The CONT group refrained from all forms of hydrotherapy throughout the experimental period. Neither group were allowed massage during this time, however, both groups were able to stretch ad libitum.

\section{Living Conditions}

Paragraph Number 7Throughout the 39-days the subjects lived onsite at the Australian Institute of Sport (AIS) in Canberra. Dietary intake was not controlled throughout the study however all participants had equal access to food and snacks as provided by the AIS dining Hall.

\section{Training details}

Paragraph Number 8All long rides were completed together as a single cohort and involved variable terrain (e.g. climbing and flats). All long rides were supervised by a follow vehicle and a coach to ensure compliance. A representative summary record from one of the cyclists (Garmin Cycling Computer) documents the duration of road rides (Figure 1). The duration of laboratory tests is also included in this Figure for completeness.

\section{Performance Measures}

Paragraph Number 9The two key performance tests $\left(2 \mathrm{xMMP}_{4 \min }\right.$ and HIIT) were used throughout the study to track changes in fatigue and performance. Efforts were performed on a commercially available air braked ergometer (Watt Bike, UK) which allowed measurement of power and cadence throughout the efforts. All interval sets were completed in groups of 67 riders staggered throughout the morning of a testing day. Testing times and groups were kept consistent throughout the duration of the study.

\section{The Interval Sets}


$2 \times 4$ min maximal test $\left(2 \times M M P_{4 m i n}\right)$ :

Paragraph Number 10The $2 \mathrm{xMMP}_{4 \min }$ test involved two, four-minute maximal efforts completed 42 minutes apart. A controlled warm-up was performed prior to each of the two efforts. The warm-up included three stages of work set at different percentages of maximum heart rate determined during the initial incremental test. The subjects were seated between the two efforts (passive recovery). This design was based on a repeated bout protocol previously used to detect overreaching and overtraining syndrome (12) and represents shortest possible time between track cycling pursuit races. The complete protocol of the $2 \mathrm{xMMP}_{4 \min }$ test is outlined in Table 2.

High Intensity Interval Training (HIIT)

Paragraph Number 11The HIIT test was designed to challenge the riders in a variety of different facets of cycling fitness. The test involved a variety of efforts including short sprints (6 sec), longer sprints (20 sec), repeat sprints, track cycling pursuit format repeat efforts and a 10 minute time trial (See Table 2 for detail). The two pursuit formatted repeat efforts were designed to mimic team pursuit performance with participants completing maximal efforts interspersed with 250W efforts. Participants completed the warm-up prior to the HIIT test on their own bike connected to stationary magnetically resisted trainers, with the HIIT test itself performed on a commercially available air braked ergometer (Watt Bike, UK).

\section{Other tests}

Incremental Test

Paragraph Number 12As previously mentioned all cyclists completed an incremental step test for the determination of MAP and peak heart rate. The test started at $100 \mathrm{~W}$ and increased 
50W every 5 minutes until the subjects either reached volitational exhaustion or could not maintain a cadence of above $60 \mathrm{rpm}$.

Set 3 Test

Paragraph Number 13The Set 3 test was another lab based interval set performed on ergometers. It involved two, four-minute maximal efforts (similar to $2 \mathrm{xMMP}_{4 \min }$ test). The purpose of the test was to add to the fatigue of the cyclists and was not used as a performance measure.

\section{Sleep Monitoring}

Paragraph Number 14Objective sleep/wake patterns were assessed using wrist activity monitors (Philips Respironics, Bend, Oregon) in conjunction with self-report sleep diaries. An activity monitor is a lightweight device worn like a wristwatch that continuously records the timing and quantity of body movement (stored in 1-min epochs for this study) with a piezo-electric accelerometer. Participants were instructed to wear the activity monitor on the same wrist at all times during the data collection period, except when showering. In the selfreport sleep diary, participants recorded sleep start and end times for all sleep periods. Data from the sleep diaries and activity monitors were used to determine when participants were awake and when they were asleep. Measures extracted from the activity monitor records and the sleep diaries included:

- $\quad$ Bedtime (hh:mm): the self-reported clock time at which a participant went to bed to attempt to sleep.

- Wake up time (hh:mm): the self-reported clock time at which a participant got out of bed and stopped attempting to sleep.

- Time in bed (h): the amount of time spent in bed attempting to sleep between bedtime 
and get-up time.

- $\quad$ Sleep onset latency ( $\mathrm{min})$ : the period of time between bedtime and sleep start.

- $\quad$ Sleep duration (h): the amount of time spent in bed asleep.

- Sleep efficiency (\%): sleep duration expressed as a percentage of time in bed.

- Mean activity score (objective sleep quality measure): an index of the magnitude of activity during sleep periods. Specifically, it is the sum of the activity counts between sleep onset time and wake up time divided by the number of epochs between sleep onset time and wake up time.

\section{Recovery Stress Questionnaire}

Paragraph Number 15Perceptual measures of stress and recovery were assessed using the recovery-stress questionnaire for athletes (RESTQ-Sport; (6)) at baseline, end of intensified training (Trial 4), and mid- taper (Trial 5). Briefly, the RESTQ-Sport is a psychometric instrument that can be used to assess an individual's recovery-stress state. It consists of 12 general scales; with seven additional sport-specific scales (totalling ten stress scales and nine recovery scales). A general indicator of the recovery-stress balance (global score) is calculated as the total stress score minus the total recovery score. Increases in the stress component represent higher subjective strain, whilst increases in the recovery component represent adequate recovery. For a detailed description of the RESTQ-Sport see Kellmann and Kallus (6).

\section{Statistics}

Paragraph Number 16Data were analyzed using a mixed linear modeling procedure (Proc Mixed) in the Statistical Analysis System (version 9.2, SAS Institute, Cary, NC). Means for 
graphical presentation were adjusted for missing values via estimation with a fixed effect specifying every level of Group (control, cold-water immersion), Trial (1 to 5) and Repetition (for performance measures), with a random effect for cyclists and allowance for only one residual error in each group across all trials. Change scores between Trial 1 (baseline) and either Trial 4 (following intensified training) or Trial 5 (mid-taper) were modeled with a covariate to adjust for any differences in baseline means between groups and with different residual error in the two treatment groups to estimate difference standard deviations of the change scores. Measures of power output were analyzed via log transformation and effects were expressed as percent changes; all other measures were analyzed via raw scores. RESTQ was converted into a theoretical 0-100 point scale.

Paragraph Number 17Uncertainty in the estimates of effects on performance was expressed as $90 \%$ confidence limits and as probabilities that the true value of the effect was beneficial, trivial or harmful in relation to threshold values for benefit and harm. A threshold of $\pm 1.0 \%$ was used for measures of power output, which is approximately 0.3 of the within-subject standard deviation a top cyclist would show in mean power between competitions in a range of events $(4,14)$. For all other measures the threshold was approximately 0.20 of the between-cyclist standard deviation in the baseline assessment. Probabilities of benefit and harm are not presented quantitatively but were used to make a qualitative probabilistic clinical inference about the effect in preference to a statistical inference based on a nullhypothesis test (4). Briefly, the effect was deemed unclear when the chance of benefit was sufficiently high to warrant use of the treatment but the risk of harm was unacceptable. Such unclear effects were identified as those with an odds ratio (OR) of benefit to harm of $<66$, a ratio that corresponds to an effect that is borderline possibly beneficial (25\% chance of benefit) and borderline most unlikely harmful (0.5\% risk of harm). All other effects were deemed clinically clear and expressed as the chance of the true effect being trivial, beneficial 
or harmful with the following scale: 25-75\%, possibly; 75-95\%, likely; 95-99.5\%, very likely; >99.5\%, most likely).

\section{RESULTS}

Paragraph Number 18Post training performance data is only included for Trial 5 (midway through taper) rather than Trial 6 (end of taper). This was necessary due to a subsequent National cycling competition immediately post Trial 6 resulting in not all subjects willing to perform maximal efforts in performance tests during Trial 6. To examine the adaptation responses of athletes to CWI or control, Trial 5 data is compared to baseline date (Trial 1). In some instances, the immediate effects of intensified training has also been analysed by comparing Trial 4 with Trial 1.

\section{Performance}

\section{$\mathbf{2 x M M P}_{4 \min }$}

Paragraph Number 19Performance assessed by $2 \mathrm{xMMP}_{4 \min }$ increased from baseline (Trial 1) to Trial 5 in both groups (CWI: 9.3\%, $\pm 3.0 \%, \mathrm{OR}=42 \times 10^{5}$; CONT: $6.5 \%, \pm 5.2 \%$, OR=1212). The CWI group was slightly greater than control $(2.7 \%, \pm 5.7 \%)$, however this difference was unclear $(\mathrm{OR}=14)$ (Figure 2a). By subtracting the power produced during the first $4 \mathrm{~min}$ bout from the power produced in the second 4min bout (4MMP2-4MMP1) an indication of repeat cycling performance can be examined. When comparing this repeat performance ability on Trial 5 compared to Trial 1, the CWI group demonstrated a $3.0 \%$, $\pm 3.8 \%$ greater increase than CONT, with an odds ratio of 90 . When examining the effects of CWI on higher level participants (participants who were 1 standard deviation above the mean on Trial 1), there was no clear effect on average 2x4MMP $(4.3 \%, \pm 8.3 \%)$. However when 
examining the same response to the 4MMP2-4MMP1 there was a very likely beneficial effect of CWI for the higher level subjects (10.8\%, $\pm 7.4 \%)$ (Figure $2 b)$.

High Intensity Interval Training (HIIT)

Paragraph Number 20Average MMP1s during Trial 5 increased from baseline in the CWI group $(2.5 \%, \pm 3.5 \%, \mathrm{OR}=66)$ and decreased in the CONT group $(-1.8 \%, \pm 2.8 \%, \mathrm{OR}=0)$. The difference between the groups was therefore $4.4 \%, \pm 4.2 \%$, with an OR of 452 (Figure 3a). There were no clear effects of CWI on performance of the higher level subjects (3.5\%, 士7.1\%) Time trial performance also increased in both groups after training and taper compared to baseline (CWI: 5.4\%, $\pm 3.7 \%$, OR=2860; CONT: 5.9\%, $\pm 2.8 \%$, OR=138X10 ${ }^{3}$ ). However, there were no clear differences between groups $(-0.4 \%, \pm 4.3 \%$; OR=1) (Figure 3b). There was also no clear effect of CWI for the subjects who performed 1 standard deviation below the mean on Trial 1 (faster time trial) $(4.3 \%, \pm 7.5 \%)$.

Paragraph Number 21The power produced during the preload on Pursuits 1 and 2 was instructed to be $250 \mathrm{~W}$. However, as subjects were asked to maintain this power and it was not enforced, the power produced during these preloads can be examined to measure 'selfselected' power. During the preload in Pursuit 1 both groups demonstrated increased power following taper when compared to baseline (CWI: 6.0\%, $\pm 2.1 \%$, OR=533X10³ ; CONT: $3.6 \%, \pm 2.5 \%, \mathrm{OR}=4251$ ), resulting in a higher self-selected power output in the CWI group (2.4\%, $\pm 3.0 \%, O R=91)$ (Figure 3c). An almost identical result was observed for the second pursuit preload (CWI: $5.2 \%, \pm 1.7 \%$, OR=106x10 ${ }^{4}$; CONT: $3.0 \%, \pm 2.3 \%$, OR=2065), again demonstrating higher power outputs in the CWI group $(2.1 \%, \pm 2.7 \%$, OR=97) (Figure 3d). The effects of CWI on the higher level subjects were unclear for both Pursuit 1 (1.1\%, $\pm 3.5 \%)$ and $2(1.3 \%, \pm 3.0 \%)$.

\section{Sleep and Perceptual Measures (RESTQ-Sport)}


Paragraph Number 22Changes from baseline to Trial 4 (end of intensified training) are examined in an attempt to understand changes that may be occurring as a result of the intensified training, prior to taper. The intensified training had a negative effect on total sleep time, sleep efficiency, mean activity score, global REST-Q and REST-Q stress scale in both the CWI and control group (Trial 4- Trial 1; Table 3). However, there were no clear effects between interventions on any of the measures, with the exception of a possibly beneficial effect of cold water immersion on the REST-Q Recovery Scale.

Paragraph Number 23 Changes in sleep and perceptual measures from baseline (Trial 1) to mid-taper (Trial 5) are reported in Table 3. Following one week of taper, total sleep time, sleep efficiency, mean activity score, global REST-Q, REST-Q stress scale and REST-Q Recovery remained negatively affected in both the CWI and control groups (Trial 5- Trial 1; Table 3). Additionally, there was a possibly harmful effect of CWI on sleep latency, which resulted in a moderately harmful difference between CWI and control following a taper period. Similarly, a possibly harmful effect of CWI was observed on all REST-Q measures at the same time period when compared to control.

\section{DISCUSSION}

Paragraph Number 24The results of this investigation suggest that cold water immersion completed four times per week over three weeks of intensified training and taper does not impair adaptation to training in competitive cyclists. In contrast, when examining repeat high intensity performance $\left(2 \mathrm{xMMP}_{4 \mathrm{~min}}\right)$, sprint performance (MMP1s) and self-selected workloads, the cold water immersion group demonstrated a greater increase in performance when compared to the control group. 
Paragraph Number 25A recent meta-analysis examining the effects of cold water immersion on recovery from strenuous exercise, reported that cold water immersion is an effective strategy to reduce muscle soreness (9). A number of other studies have also demonstrated an acute positive effect of cold water immersion on performance in cycling, running and team sports $(1,7,18-20)$. However, the use of habitual or chronic hydrotherapy has recently been questioned and suggested to be potentially negative to long-term adaptation to training (24). Although the exact mechanisms underlying both cold water immersion and adaptation to training are not clear, it has been suggested that reducing fatigue and/or inflammation post exercise may be involved.

Paragraph Number 26 The findings of the current study showed that there was a greater change in average sprint power (4.4\%), repeat cycling performance (3.0\%) and a trend for increased power in the $2 \mathrm{xMMP}_{4 \min }(2.7 \%)$ over the training period. In contrast, Yamane et al (24), reported that regular cold water immersion of the dominant limb following exercise over a 4-6 week training period resulted in an attenuated increase in cycling performance relative to the non-immersed limb (16\% in control leg vs. $9 \%$ in the immersed leg). Handgrip exercise performance was also attenuated in the cold water immersion group. The authors concluded that micro damage and metabolic alterations may be negatively influenced by cold water immersion. Additionally, similar findings were reported by Higgins et al. (3) whereby regular cold water immersion decreased repeat sprint test performance (-0.62 ES) when compared with control in amateur rugby union players. There are however, a number of methodological concerns regarding the aforementioned papers. In the investigation by Yamane et al (24), subjects were untrained, subject numbers were low ( $\mathrm{n}=6$ for cycling exercise), water temperatures were low $\left(5^{\circ} \mathrm{C}\right.$ for leg immersion and $10^{\circ} \mathrm{C}$ for forearm immersion), immersion durations were very high ( 2 X 20 minutes for leg immersion and $1 \mathrm{X}$ $20 \mathrm{~min}$ for forearm immersion), and performance tests utilised were not representative of 
real-life athletic performance (incremental test to fatigue for cycling and sub maximal handgrip exercise to fatigue). Furthermore, there appeared to be potential for substantial human error in the test results of Higgins et al. (3) due to the methodology used (i.e. hand timed sprint test, total sprint distance was approximated).

Paragraph Number 27Howatson et al (5) examined the influence of cold water immersion on maximum voluntary contraction, perception of muscle soreness, creatine kinase, muscle girths and range of motion following two bouts of drop jump exercise separated by 14-21 days. Subjects received either cold water immersion $\left(12 \mathrm{~min}\right.$ at $\left.15^{\circ} \mathrm{C}\right)$ or seated rest after the first bout immediately post exercise, $24 \mathrm{hr}, 48 \mathrm{~h}$ and $72 \mathrm{~h}$ post exercise. No treatment was provided after the second bout. Results demonstrated no significant differences in any of the variables measured when comparing the cold water immersion group to the control group, indicating no effect either positive or negative on adaptation to training. However, this was only acute use of the intervention, with only one exposure to CWI. It is possible that more prolonged use may have induced an effect on adaptation.

Paragraph Number 28Finally, some studies have examined the use of ice or cold packs post exercise on aspects of muscle recovery. While ice/cold packs may have significant differences to cold water immersion in both performance outcomes and mechanism of action (11), the results of these papers have been used to question the role of hydrotherapy in adaptation to training. Nemet et al (13) exposed 12 elite junior handball players to 2 x 15 min cold pack application to the legs immediately following 4 x 250m running efforts. Cold pack application resulted in significant decreases in Interleukin-1 $\beta$, Interleukin-1ra, Insulin-like Growth Factor-1 and Insulin-like growth factor-binding protein (IGFBP)-3 and a greater increase in IGFBP-1 during recovery. The authors concluded that local ice therapy resulted in 
a greater decrease of both pro- and anti-inflammatory cytokines and a greater decrease in anabolic hormones (13). This was primarily attributed to muscle hypothermia in the immersion trials. It was suggested that muscle hypothermia may have interfered with myofibre regeneration and thus was harmful to the adaptation process (24). This study has also been referred to to suggest that recovery strategies may impair the adaptation process. However, unlike the present investigation, the previous study investigated the effects of the cold application on only one occasion and therefore any implications for the adaptation process should be interpreted with caution.

Paragraph Number 29The difference between the positive results observed in the current study in comparison to previous research may be related to the higher training status of the current participants, the use of a more appropriate cold water immersion temperature, the use of CWI rather than cold pack application and/or the ecologically valid performance assessments.

Paragraph Number 30With the exception of sleep latency, all measures of sleep were affected in a negative manner at the end of the intensified training period, regardless of intervention. Further, following taper total sleep time and sleep latency were negatively influenced by CWI. Although there were differences between CWI and control of two and five minutes for total sleep time and sleep latency, respectively; the clinically relevant smallest worthwhile change for these measures is currently unknown.

Paragraph Number 31At present, there is very little scientific evidence investigating the effects of intensified training on sleep. Taylor et al. investigated sleep changes in seven national level female swimmers across a competitive season, including taper period (17). 
While no change in sleep onset latency, time awake after sleep onset, total sleep time and rapid eye movement (REM) sleep were observed throughout the training period, the number of movements during sleep during the high volume training phase was significantly higher than during the taper period (11\%). Increased muscle soreness and muscle fatigue was provided as a possible explanation for the additional sleep disturbance (17).

Paragraph Number 32Intensified training with or without CWI negatively influenced global REST-Q and REST-Q Stress. As expected, Rest-Q Recovery scores were also negatively influenced in the control group, but not the CWI group, resulting in the CWI group subjectively reporting higher perceptions of recovery than the control group. The interactions were notably different after the 6d taper, with possibly harmful effects of CWI on all RESTQ measures.

Paragraph Number 33Interestingly, in the present study, while most aspects of sleep and mood was negatively influenced at the end of intensified training and taper in both CWI and control, performance continued to increase. This finding reflects anecdotal reports from elite coaches who describe that competitive athletes may experience both mood and sleep disturbance and still improve high intensity, short-duration performance. It should be noted that results of the current study pertain to a three week intensified training period and therefore may not be relevant to longer periods of training. Further, much of the previous training and/or overreaching research have not utilised highly trained subjects. The current study demonstrates that it may be difficult to fatigue trained cyclists sufficiently to result in a decrease in performance over a 3 week period.

Paragraph Number 34Immersion in water results in a number of physiological changes which have been investigated in a bid to identify potential mechanisms for increased performance with acute recovery. From a cardiovascular perspective, research has identified 
increases in central blood volume, cardiac volume, heart rate and decreased peripheral resistance as a result of hydrostatic pressure causing a redistribution of blood flow (21). Cold stimulus results in compensatory mechanisms to minimise heat loss, such as peripheral vasoconstriction, which is regionally dependent (8). Hydrostatic pressure may also limit the formation of oedema (22) and assist in reducing existing muscle oedema by causing fluid to move from the interstitial to the intravascular space for clearance. Decreases in tissue temperature have also been found to decrease nerve conduction velocity therefore decreasing pain perception (analgesia) and reducing muscle spasm (11). Numerous studies have also reported reductions in skin, muscle and core temperature as a result of CWI $(2,15)$.

Paragraph Number 35While a number of potential mechanisms exist which may explain the acute benefits of CWI on performance, there is very minimal information regarding the means by which CWI may enhance long term performance. While the theory that a less fatigued athlete should be able to complete training at an increased quality and quantity holds potential merit, in the present investigation all athletes trained as a group. From the performance testing the subjects completed more work in laboratory ergometer training sessions, however it is questionable as to whether this high intensity component of training was the primary driver of the increased performance observed during the repeated $\mathrm{MMP}_{4 \mathrm{~min}}$ efforts.

Paragraph Number 36Research has recently examined the molecular mechanisms involved in adaptation to training. The transcriptional coactivator peroxisome proliferators-activator receptor gamma coactivator-1 alpha (PGC-1 $\alpha$ ) has been shown to be an important regulator of mitochondrial function, oxidative metabolism and energy homeostasis (10). PGC-1 $\alpha$ expression is induced following acute exercise and this has been shown to improve performance (23). Interestingly, PGC-1 $\alpha$ expression is also temperature sensitive, with evidence in animals that exposure to cold $\left(4^{\circ} \mathrm{C}\right)$ results in increased expression in brown fat 
and muscle. More recently, acute exposure to a cold environment $\left(7^{\circ} \mathrm{C}\right)$ in humans was shown to result in a larger increase in PGC-1 $\alpha$ when compared to room temperature (16). While PGC-1 $\alpha$ expression was not measured in this study, it represents a possible mechanism for the enhanced response to training following chronic CWI exposure and may be an important area of future research.

Paragraph Number 37 Finally, it is important to mention that this was a cross-sectional study that was not blinded. Although subjects were matched for both belief in recovery as well as fitness, it is possible that the CWI group experienced a positive effect associated with being treated, in addition to the effects of the treatment itself. However, this study was aimed to represent a real-world scenario, reflecting that athletes either do or do not engage in this form of recovery during training. As with many real-world research designs, a lack of placebo may confound the elucidation of the mechanisms associated with the observed benefits.

Paragraph Number 38The primary objective of this study was to evaluate whether cold water immersion during a 3-week phase of rigorous cycling training (simulating aspects of a Grand Tour) would impair cycling performance. In summary, data from this study do not support recent speculation that cold-water immersion is detrimental to adaptations to 3-weeks of increased training load in competitive cyclists. Future research is needed to identify the mechanisms by which this may occur and to examine whether adaptation is influenced by cold water immersion in sports other than cycling. 
The authors would like to thanks all the cyclists who participated in this study.

This investigation was supported by funding from the Australian Sports Commission and the Australian Research Council.

The authors declare that there are no conflicts of interest in undertaking this study.

Results of the present study do not constitute endorsement by the American College of Sports Medicine. 
List of Tables

Table 1 Schedule of laboratory testing and road riding. Inc= Incremental Test, HIIT =high intensity interval test, Famil= familiarisation session, $2 \mathrm{xMMP}_{4 \min }=2 \mathrm{x} 4 \mathrm{~min}$ test, Comp=National Series Competition. Times in italics indicate road ride durations.

Table 2 Description of 2x4 minute maximal test and High Intensity Interval Test. HRmax= Maximum heart rate. Max= Maximum voluntary effort, $\mathrm{W}=$ Watts.

Table 3 Changes in measures of sleep and recovery-stress (from the REST-Q) from Trial 1 (baseline) to Trial 4 (following intensified training) and Trial 5 (mid-taper). 
List of Figures

Figure 1 A representative summary for a single subject showing the duration of road rides and laboratory tests.

Figure 2 Performance as assessed by the $2 x 4$ min maximal test $\left(2 x M M P_{4 m i n}\right)$ over the study period.

2a: Combined mean power (W). $\boldsymbol{\square}=\mathrm{CWI}, \mathrm{O}=\mathrm{CONTROL}$. Symbols with error bars represent mean and standard deviation across the 5 trials. CWI= Cold Water Immersion, $\mathrm{W}=\mathrm{Watts} . \mathrm{B}=$ Baseline, ITP= Intensified Training.

2b: Repeat performance ability as expressed by the change in 4min tests (4mMMP24mMMP1). $\boldsymbol{\square}=$ CWI, $\circ=$ CONTROL. CWI $=$ Cold Water Immersion, W=Watts. $\mathrm{B}=$ Baseline, ITP= Intensified Training.

Figure 3 Performance assessed during the High Intensity Interval Test (HIIT) over the training period.

3a: Peak sprint power (W). $\mathbf{\square}=\mathrm{CWI}, \mathrm{O}=\mathrm{CONTROL}$. Symbols with error bars represent mean and standard deviation across the 5 trials. $\mathrm{CWI}=$ Cold Water Immersion, $\mathrm{W}=\mathrm{Watts} . \mathrm{B}=$ Baseline, ITP= Intensified Training.

3b: Mean power $(\mathrm{W})$ during the 10 minute Time Trial $(10 \mathrm{minTT}) \boldsymbol{\square}=\mathrm{CWI}, \circ=\mathrm{CONTROL}$. CWI= Cold Water Immersion, W=Watts. B= Baseline, ITP= Intensified Training.

3c: Pursuit 1 (W). $\mathbf{\square}=$ CWI, $\circ=$ CONTROL. Symbols with error bars represent mean and standard deviation across the 5 trials. CWI= Cold Water Immersion, $\mathrm{W}=\mathrm{Watts} . \mathrm{B}=\mathrm{Baseline}$, ITP= Intensified Training. 
3d: Pursuit 2 (W) $\mathbf{\square}=$ CWI, o = CONTROL. Symbols with error bars represent mean and standard deviation across the 5 trials. CWI= Cold Water Immersion, $\mathrm{W}=\mathrm{Watts} . \mathrm{B}=\mathrm{Baseline}$, ITP= Intensified Training. 


\section{References}

1. Bailey DM, Erith SJ, Griffin PJ, Dowson A, Brewer DS, Gant N, and Williams C. Influence of cold-water immersion on indices of muscle damage following prolonged intermittent shuttle running. J Sports Sci. 2007;25(11):1163-70.

2. Gregson W, Black MA, Jones H, Milson J, Morton J, Dawson B, Atkinson G, and Green DJ. Influence of cold water immersion on limb and cutaneous blood flow at rest. Am J Sports Med. 2011;39(6):1316-23.

3. Higgins TR, Heazlewood IT, and Climstein M. A random control trial of contrast baths and ice baths for recovery during competition in U/20 rugby union. $J$ Strength Cond Res. 2011;25(4):1046-51.

4. Hopkins WG, Marshall SW, Batterham AM, and Hanin J. Progressive statistics for studies in sports medicine and exercise science. Med Sci Sports Exerc. 2009;41(1):3-13.

5. Howatson G, Goodall S, and van Someren KA. The influence of cold water immersions on adaptation following a single bout of damaging exercise. Eur J Appl Physiol.

2009;105(4):615-21.

6. Kellmann M, and Kallus KW. The recovery-stress-questionnaire for athletes. South Australia: Human Kinetics; 2002, p. 3-301.

7. King M, and Duffield R. The effects of recovery interventions on consecutive days of intermittent sprint exercise. J Strength Cond Res. 2009;23(6):1795-802.

8. Lee DT, Toner MM, McArdle WD, Vrabas IS, and Pandolf KB. Thermal and metabolic responses to cold-water immersion at knee, hip, and shoulder levels. J Appl Physiol. 1997;82(5):1523-30.

9. Leeder J, Gissane C, van Someren K, Gregson W, and Howatson G. Cold water immersion and recovery from strenuous exercise: a meta-analysis. Br J Sports Med. 2012;46(4):233-40.

10. Lin J, Handschin C, and Spiegelman BM. Metabolic control through the PGC-1 family of transcription coactivators. Cell Metab. 2005;1(6):361-70.

11. Meeusen R, and Lievens P. The use of cryotherapy in sports injuries. Sports Med. 1986;3(6):398-414.

12. Meeusen R, Nederhof E, Buyse L, Roelands B, de Schutter G, and Piacentini MF. Diagnosing overtraining in athletes using the two-bout exercise protocol. Br J Sports Med. 2008;44(9):642-8.

13. Nemet D, Meckel Y, Bar-Sela S, Zaldivar F, Cooper DM, and Eliakim A. Effect of local cold-pack application on systemic anabolic and inflammatory response to sprint-interval training: a prospective comparative trial. Eur J Appl Physiol. 2009;107(4):411-7.

14. Paton CD, and Hopkins W. Variation in performance of elite cyclists from race to race. European Journal of Sport Science. 2009;6:1-7.

15. Peiffer JJ, Abbiss CR, Watson G, Nosaka K, and Laursen PB. Effect of cold-water immersion duration on body temperature and muscle function. J Sports Sci. 2009;27(10):98793.

16. Slivka DR, Dumke CL, Tucker TJ, Cuddy JS, and Ruby B. Human mRNA response to exercise and temperature. Int J Sports Med. 2012;33(2):94-100.

17. Taylor SR, Rogers GG, and Driver HS. Effects of training volume on sleep, psychological, and selected physiological profiles of elite female swimmers. Med Sci Sports Exerc. 1997;29(5):688-93.

18. Vaile J, Halson S, Gill N, and Dawson B. Effect of cold water immersion on repeat cycling performance and thermoregulation in the heat. J Sports Sci. 2008;26(5):431-40. 19. Vaile J, Halson S, Gill N, and Dawson B. Effect of hydrotherapy on recovery from fatigue. Int J Sports Med. 2008;29(7):539-44. 
20. Vaile J, Halson S, Gill N, and Dawson B. Effect of hydrotherapy on the signs and symptoms of delayed onset muscle soreness. Eur J Appl Physiol. 2008;102(4):447-55.

21. Wilcock IM, Cronin JB, and Hing WA. Physiological response to water immersion: a method for sport recovery? Sports Med. 2006;36(9):747-65.

22. Wilcock IM, Cronin JB, and Hing WA. Physiological response to water immersion: a method for sport recovery? Sports Med. 2006;36(9):747-65.

23. Wu J, Ruas JL, Estall JL, Rasbach KA, Choi JH, Ye L, Bostrom P, Tyra HM, Crawford RW, Campbell KP, Rutkowski DT, Kaufman RJ, and Spiegelman BM. The unfolded protein response mediates adaptation to exercise in skeletal muscle through a PGC-

1alpha/ATF6alpha complex. Cell Metab. 2011;13(2):160-9.

24. Yamane M, Teruya H, Nakano M, Ogai R, Ohnishi N, and Kosaka M. Post-exercise leg and forearm flexor muscle cooling in humans attenuates endurance and resistance training effects on muscle performance and on circulatory adaptation. Eur J Appl Physiol.

2006;96(5):572-80. 